Sur le terrain de

l'océanographie

politique : carnets de terrain,

ethnographie multi-sites et

modes de gouvernement de la

Mer au croisement des sciences

sociales et des sciences de la

nature

\title{
Anatole Danto
}

Laboratoire des Sciences de l'Environnement Marin et Centre de Recherches sur l'Action Politique en Europe, France

\section{Camille Mazé}

Laboratoire des Sciences de l'Environnement Marin et Centre de Recherches sur l'Action Politique en Europe, France

\section{Olivier Ragueneau}

Laboratoire des Sciences de l'Environnement Marin, France

\section{Résumé}

Cet article présente une partie des « carnets de terrain » des membres du groupe ApoliMer (Anthropologie politique de la Mer / Approche éco-politiste de la mer). Ce réseau thématique pluridisciplinaire international (RTPi) du CNRS s'intéresse aux modes de gouvernement de la mer, en combinant sciences sociales du politique, sciences de la nature et sciences de l'ingénieur. Tout au long du texte, une partie des matériaux ethnographiques issus des enquêtes de terrain de l'équipe basée au Laboratoire des sciences de l'environnement marin (LEMAR) à Brest, est mobilisée pour décrire la manière dont les chercheurs décryptent les formes de gouvernement des terrains maritimes et littoraux, dans une étroite interdisciplinarité. Cet article est

\section{Corresponding author:}

Anatole Danto, LEMAR (UMR 6539), Laboratoire des Sciences de l'Environnement Marin, Institut Universitaire Européen de la Mer, Technopôle Brest-Iroise, 29280 Plouzané, France.

Email: anatole.danto@orange.fr 
l'occasion d'exposer la genèse et l'évolution du RTPi, au travers de différentes "saisons" de recherches ayant marqué son existence, depuis sa naissance en 20I3-20I4.

\title{
Mots-clés
}

anthropologie politique, ethnographie multi-site, gouvernement de la mer, interdisciplinarité, océanographie politique

\begin{abstract}
This article presents a part of the 'Field notebooks' of the members of the International Multidisciplinary Thematic Network (RTPi) of CNRS ApoliMer (political anthropology of the sea), which focuses on the form of government of the sea, at the crossroads of the social sciences policy and natural sciences. Throughout the text, some of the ethnographic materials from the team's fieldwork surveys are used to describe how ApoliMer tracks governance on marine and coastal lands in an interdisciplinary manner. This article is also an opportunity to expose the genesis and evolution of the RTPi, through the various 'seasons' of research that has marked its existence since its birth in 2013-2014.
\end{abstract}

\section{Keywords}

government of the sea, interdisciplinarity, multi-sited ethnography, oceanography policy, political anthropology

\section{Nouvelles vagues - suivre « la gouvernance de la mer »}

Dans cet article, nous partageons les premiers extraits des carnets de terrain d'ApoliMer.[1] ApoliMer est un réseau de recherche international pluridisciplinaire qui explore les enjeux et les modalités concrètes du gouvernement de la mer, en combinant sciences sociales (science politique, sociologie, anthropologie, histoire, géographie, droit), sciences de la nature (biologie, biogéochimie, écologie) et sciences de l'ingénieur (ingénierie écologique).

L'axe prioritaire de recherche d'ApoliMer consiste à rentrer dans la boîte noire du système de décision publique et d'action collective autour de « la gouvernance de la mer ». Cette approche implique d'aller à la rencontre des acteurs, des groupes, des institutions qui encouragent, réalisent ou bloquent la transformation écologique et sociale dans une perspective dite durable ou soutenable. La focale est mise sur les enjeux stratégiques liés à « la gouvernance de la mer », de la zone côtière à la haute mer, dans le contexte du changement global. Notre terrain, c'est la fabrique des formes de gouvernement de la mer elle-même; il ne correspond ni à un lieu précis, stricto sensu, ni à un échelon de décision, mais se caractérise par une multiplicité de sites, ainsi que par une dynamique des jeux d'échelle et des processus en cours; il ne s'agit pas non plus d'une communauté, mais de l'étude de la représentation des communautés côtières dans les processus de 


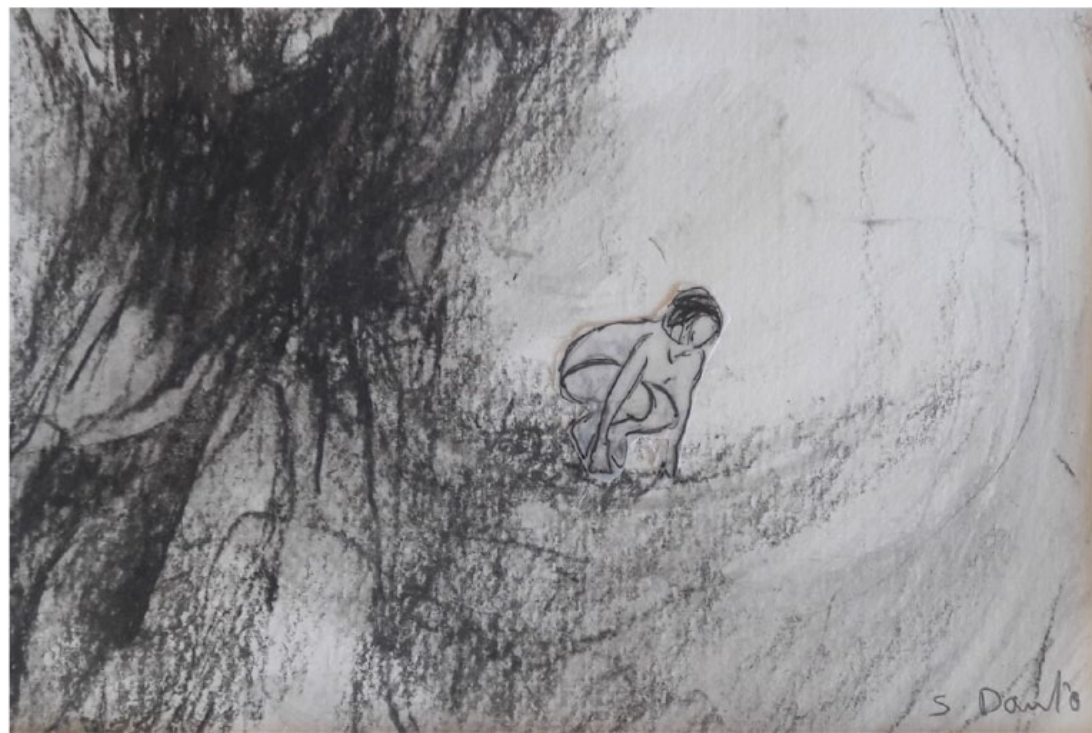

Image I. Relation Homme / Nature. Fusain, 2013. Crédit : () Sylvie Allais-Danto.

décision concernant la gestion de leur environnement; ce n'est pas non plus une institution, étudiable en soi et pour soi, fonctionnant en vase-clos, mais l'ensemble des arènes de décision, connectées entre elles; enfin, ce n'est ni un secteur, ni un acteur mais les groupes sociaux et socio-économiques, l'ensemble des individus qui les composent et les interactions entre eux, les réseaux qui font « la gouvernance de la mer ».

La démarche d'enquête implique une approche multi-site (Marcus, 1995) et multiscalaire qui permet de croiser différentes cosmogonies - autant de façons d'appréhender les relations Homme / Nature (voir image 1) et, très précisément, les formes de gouvernement de la mer par les hommes face au changement global. La question du politique (Adelle et Weiland, 2012) et des relations de pouvoir dans l'établissement des formes de contrôle, d'accès aux territoires marins et aux ressources, les frontières et la production sociale du droit sont au cœur de nos préoccupations en sciences sociales,[2] systématiquement croisées avec les savoirs des sciences de la nature (Mooney, Duraiappah, Larigauderie, 2013).

En enquêtant sur la manière dont sont gouvernés ce qu'il est désormais devenu commun de nommer les « socio-écosystèmes "[3] et sur les blocages à la mise en œuvre de leur gestion soutenable pourtant largement invoquée (cf. Objectif développement durable n. 14 de l'ONU, ODD 14 : Vie sous-marine), nous produisons une ethnographie des interstices de l'insoutenable, saisissons l'invisible ou l'intangible, en naviguant parmi les gens de mer et ceux qui la régissent. Tout l'enjeu est de mettre au jour, à partir de l'empirique, et non sur la base de déclaration d'intention, de textes normatifs ou d'injonction, les relations de pouvoir qui entourent les tensions entre exploitation et conservation des mers. Pour ce faire, il convient de saisir les instruments et les acteurs de la régulation, les actions de surveillance et de contrôle, les interactions entre Etats, nouveaux acteurs politiques et 


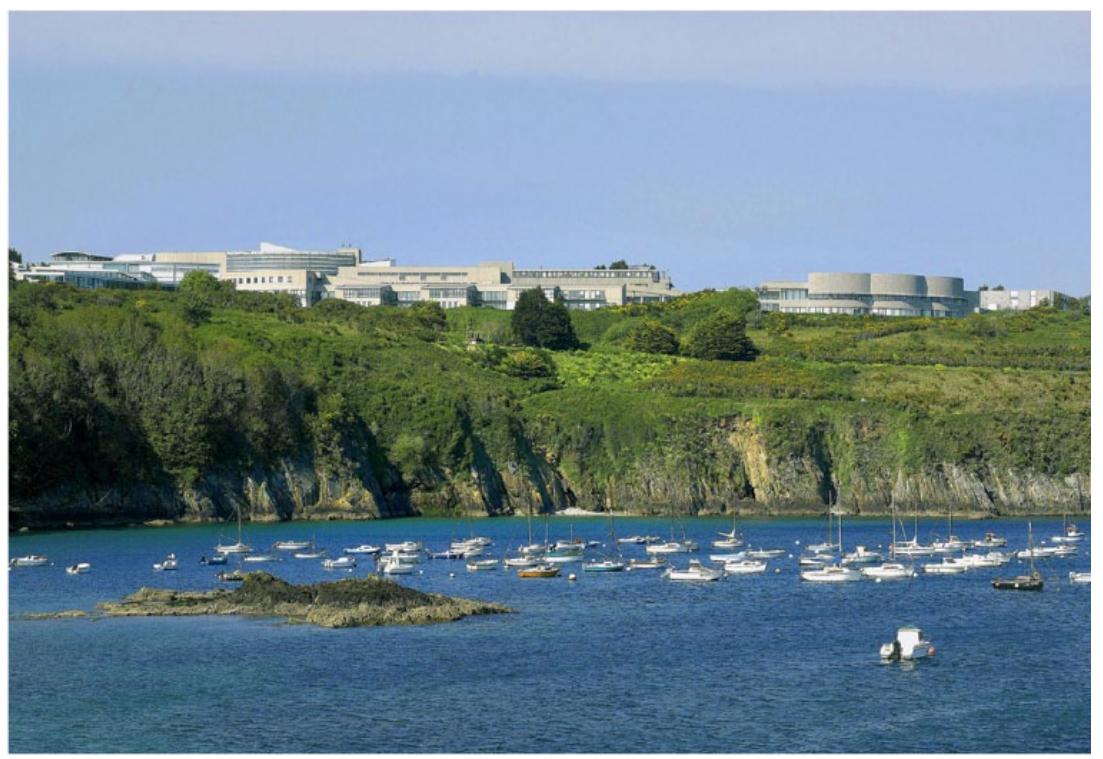

Image 2. IUEM vu de la rade.

Crédits : UBO / P. Tréguer.

communautés autour de l'accès et de la propriété des territoires et des ressources. C'est un défi immense, tant le terrain est mouvant, réticulaire, liquide. Et c'est toute la difficulté des enquêtes ethnographiques que nous menons et que nous nous attachons à ancrer dans la profondeur du terrain pour qu'elles ne soient pas « flottantes ».

La stratégie principale a consisté à immerger le camp de base d'ApoliMer, de manière durable dans une partie de son propre terrain : le lieu de production des connaissances scientifiques sur l'environnement marin.

\section{Le camp de base : la Zone Atelier Brest-Iroise (ZABrl)}

En 2013-2014, ApoliMer s'implante en surplomb du goulet de Brest, dans le Finistère breton, à l'Institut universitaire européen de la mer de l'Université de Bretagne occidentale. Le groupe est coordonné par un binôme interdisciplinaire, une politiste, anthropologue et sociologue et un biogéochimiste, sociologue des sciences, relevant du laboratoire interdisciplinaire consacré à l'environnement marin, le LEMAR. Cette unité, située à proximité de laboratoires de sciences de la nature (physique, microbiologie, géoscience) et de sciences humaines et sociales (géographie, droit, économie), couvre les champs de l'écologie, de la biologie, de la chimie, de la biogéochimie et aussi du droit. Nous sommes à quelques encablures de l'Ifremer et de l'Institut polaire français Paul Emile Victor (voir image 2).

Ce camp de base constitue notre ancrage, situé à la fois dans le paysage d'une recherche de haut vol consacrée à la mer et dans un espace géographique marqué par la présence du continuum terre-mer-atmosphère. Sous nos yeux, toute la complexité et la 


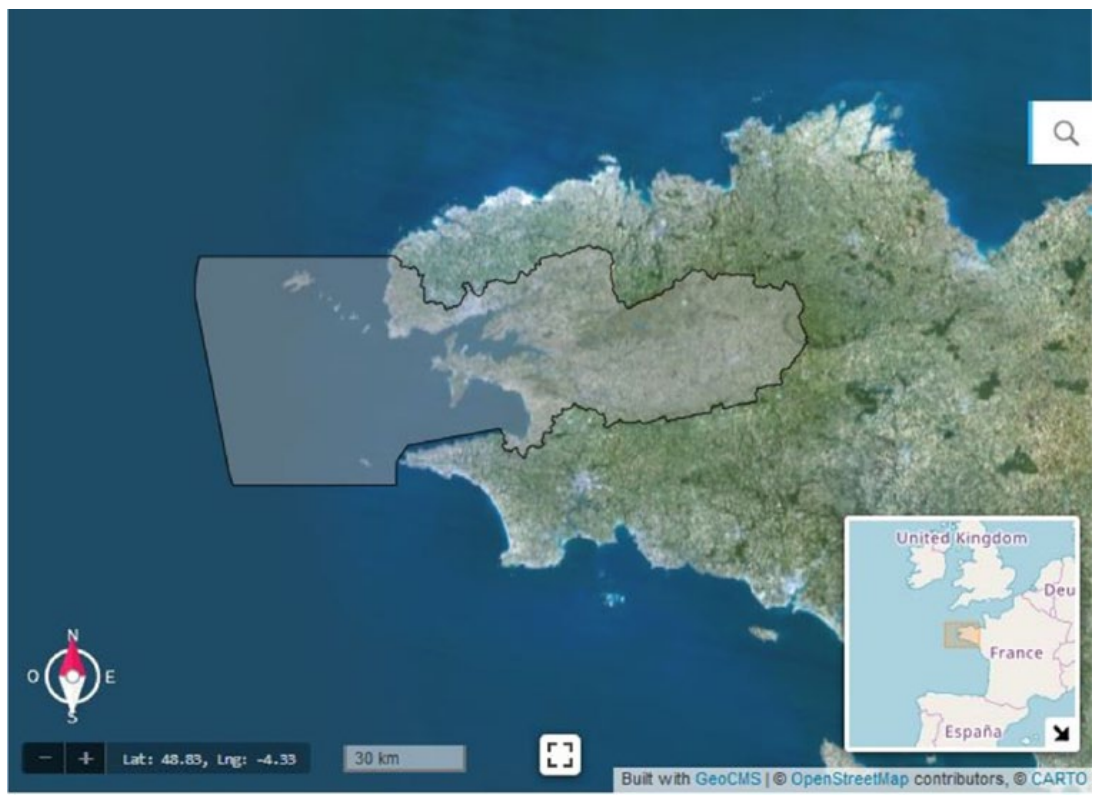

Image 3. Carte de la Zone-atelier Brest Iroise (ZABrl). Crédit : OpenStreetMap / Carto.

profondeur du terrain maritime et marin, sur lequel nous déployons une océanographie politique sur différents sites et à plusieurs échelons et notamment autour de la rade de Brest et de la mer d'Iroise (voir image 3).

ApoliMer apporte dans ce champ scientifique déjà très riche du point de vue de l'interdisciplinarité (Christie, 2011), la dimension politique (science politique, anthropologie politique, politicial ecology) et l'analyse des relations entre savoir, pouvoir et économie autour du gouvernement de la mer. Pour ce faire, le réseau ApoliMer met en interaction des chercheurs des sciences humaines et sociales et des sciences de la nature des quatre coins de la planète bleue, dont le dénominateur commun est d'appréhender la mer sous l'angle du politique.

\section{Saison I - La genèse : au cœur du socio-écosystème rade de Brest / mer d'Iroise (zone atelier Brest Iroise, ZABrl)}

Sur le terrain de la ZABrI, les membres fondateurs d'ApoliMer se sont initialement consacrés à l'interface science / politique (Jasanoff, 1990) dans le cas de la gestion du continuum terre-mer qui pose un véritable problème de «gouvernance ». Les proliférations d'algues vertes (baie de Douarnenez) ou de micro-algues produisant des toxines (ASP par exemple, Amnesic Shellfish Poisoning) qui affectent les filières pêche et aquaculture constituent un sujet de prédilection du groupe de recherche local d'ApoliMer (Raqueneau et al., 2018). L'eutrophisation représente un problème public, écologique et socioéconomique d'ordre mondial pris dans un étroit enchevêtrement de jeux d'échelle. Cette 


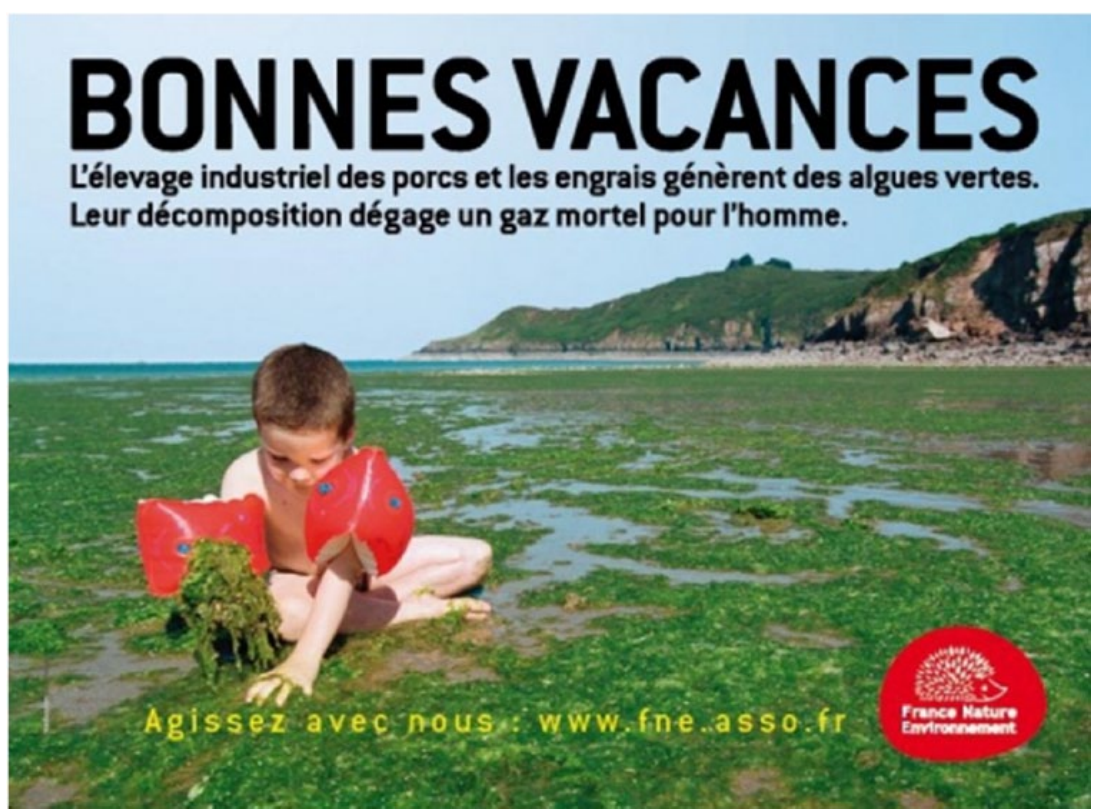

Image 4. Campagne de publicité France Nature Environnement $20 \mathrm{II}$.

Crédit : France Nature Environnement.

entrée sur le terrain du terre-mer, l'analyse du traitement public et collectif de l'impact des activités humaines et agricoles sur l'environnement et la biodiversité marine, permet le refroidissement théorique nécessaire pour saisir la crise écologique et sociale contemporaine de manière tangible dont l'extension globale et durable du phénomène d'eutrophisation constitue un révélateur, en regard de la prolifération des références à la soi-disant soutenabilité (Kates, 2011) des socio-écosystèmes côtiers et marins. L'eutrophisation permet ainsi d'aborder les relations entre savoir (science, connaissances profanes, hybridation), pouvoir (décision, politique, autorité) et avoir (marché, économie, industrie) particulièrement épineuses le long du continuum terre-mer en raison de la très forte pression anthropique et de sa vulnérabilité face au changement global (voir images 4 et 5 ).

L'étude de ces interactions ne saurait s'analyser sans se rendre de Brest à Quimper (Préfecture), Rennes (Région), Paris (Etat) et Bruxelles (Union européenne), ou encore New York (ONU) (voir images 6 et 7).

Nous baignons, au quotidien, dans les laboratoires où sont produites les connaissances dont nous suivons le circuit vers la sphère de la décision et en particulier, le LEMAR (voir images 8 et 9). Nous consignons nos observations et interprétations dans nos carnets de terrain, dont nous voulons ici livrer quelques bribes, qui nous conduisent de Brest aux Pôles.

Son 1 : Niels Petter Molvær, Solid ether, 2000.

https://www.youtube.com/watch?v=E2GQbt_OL-Y 


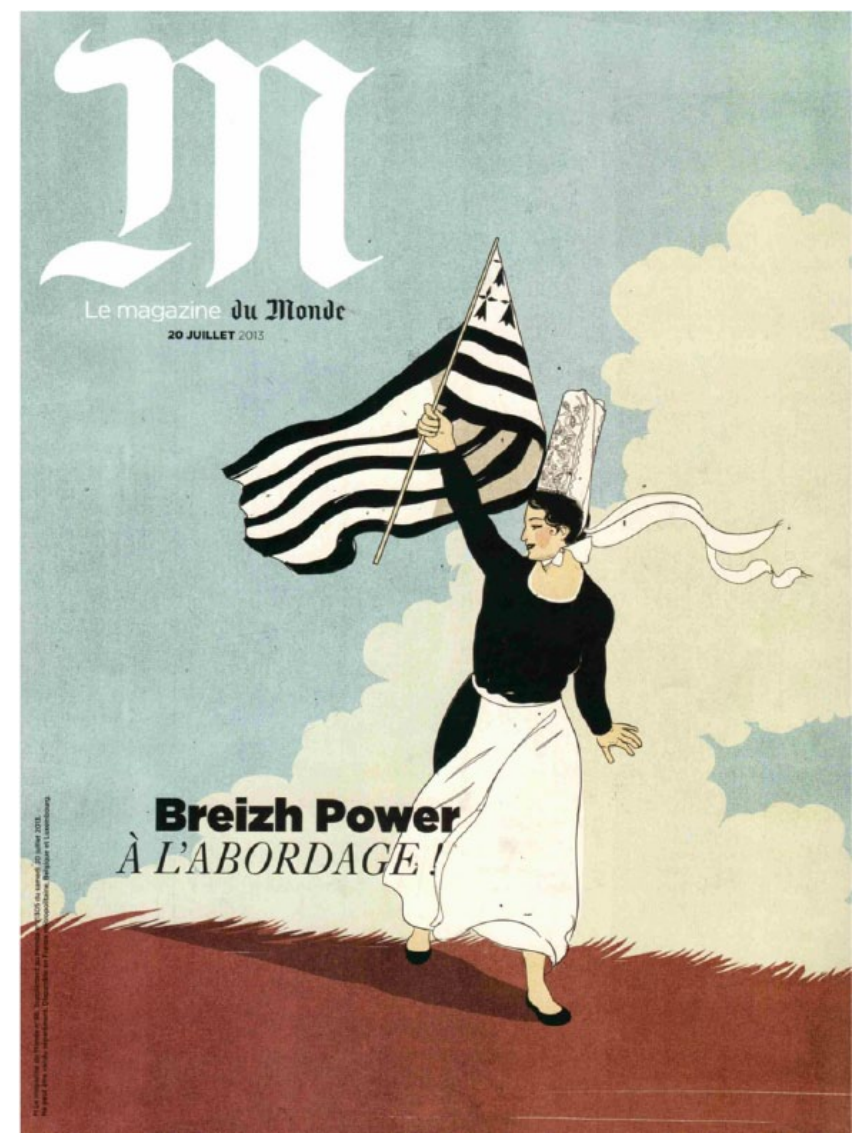

Image 5. Couverture du magazine du Monde. 20 Juillet 2013.

Credit : Journal Le Monde.

De là, certains membres de l'équipe du LEMAR embarqués dans ApoliMer, ont réalisé des missions exploratoires en Amérique du Nord (Californie, baie de San Francisco) et en Chine (Qingdao, baie de Jiaozhou) avec un regard comparatif avec la situation en Union européenne (ZABrI) pour démêler les liens entre science et politique dans ces trois contextes. Des extraits de carnets de terrain de ces missions sont ici présentés. $\mathrm{Au}$ fil des ans, les chercheurs d'ApoliMer ont mené, mènent, et envisagent d'autres projets de recherche sur les mers du globe, guidés par les anciennes grandes expéditions de l'Académie de Marine[5] qui quittèrent Brest les siècles passés à destination de contrées lointaines (voir images 10 et 11).

Son 2 : Terje Isungset et Lena Nymark, Glacier recording, 2014.

https://www.youtube.com/watch?v=QosfSaE0q7c\&list=RDwno0YKp1bGQ\&index=4 


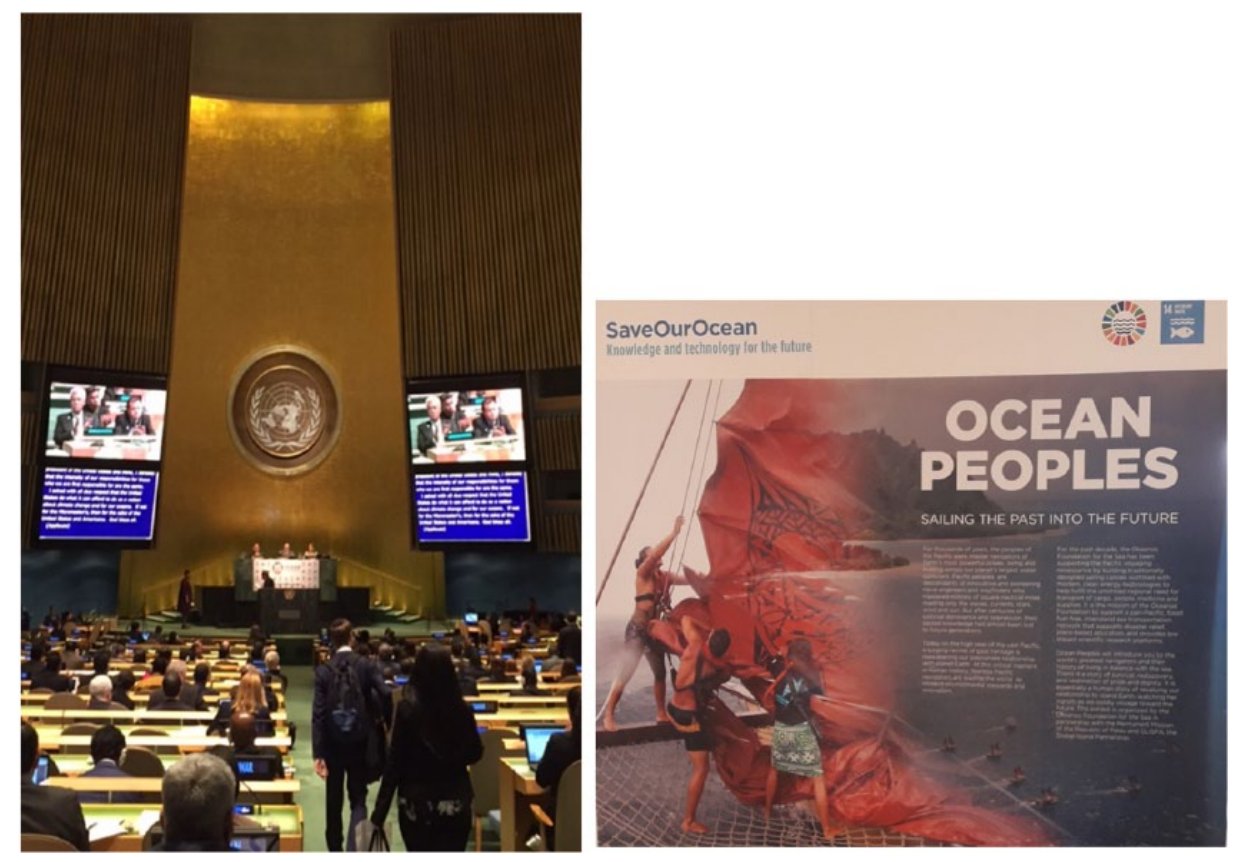

Images 6 et 7. Assemblée générale des Nations-Unies, Conférence mondiale sur l'Océan (ODD 14) juin 2017.

Crédit : Camille Mazé.

\section{Saison 2 - Passer à l'ouest : la baie de San Francisco}

Carnets de terrain, automne 2013 et septembre 20/4, Baie de San Francisco, Californie, USA. «A l'automne 2013 et en septembre 2014, nous nous rendons à deux reprises à San Francisco, en binôme. O.R. biogéochimiste et C.M., politiste. Nous naviguons en ces fins d'été, d'un laboratoire à l'autre, d'un côté à l'autre de la baie, pour comprendre la controverse qui divise les scientifiques qui travaillent sur le fonctionnement de la baie de San Francisco et en particulier sur le premier maillon de la chaîne alimentaire, le phytoplancton. Le problème se pose de mesurer et de contrôler l'équilibre entre les diatomées, qui sont considérées en écologie comme étant à la base d'une chaîne alimentaire productive et saine et d'autres espèces de phytoplancton, comme les dinoflagellés, dont un grand nombre d'espèces sont toxiques (ex. Alexandrium minutum). Entre laboratoires et équipes de recherche travaillant sur la qualité de l'eau de la baie de San Francisco, deux visions s'affrontent. Pour les uns (équipe du biogéochimiste Richard C. Dugdale au Romberg Tiburon Center (RTC) à l'Université de San Francisco (SFSU)), la qualité et la quantité de celui-ci dépendrait de la ressource en nutriments (différentes formes d'azote, nitrates NO3 et / ou ammonium NH4). Pour les autres (équipe de l'écologue Jim E. Cloern, USGS), celles-ci seraient davantage liées au contrôle de la production primaire et de la biodiversité phytoplanctonique, par un prédateur : un mollusque de l'ordre des Myoida, le Potamocorbula amurensis, qui prolifère dans la baie depuis les années 1980, prenant 

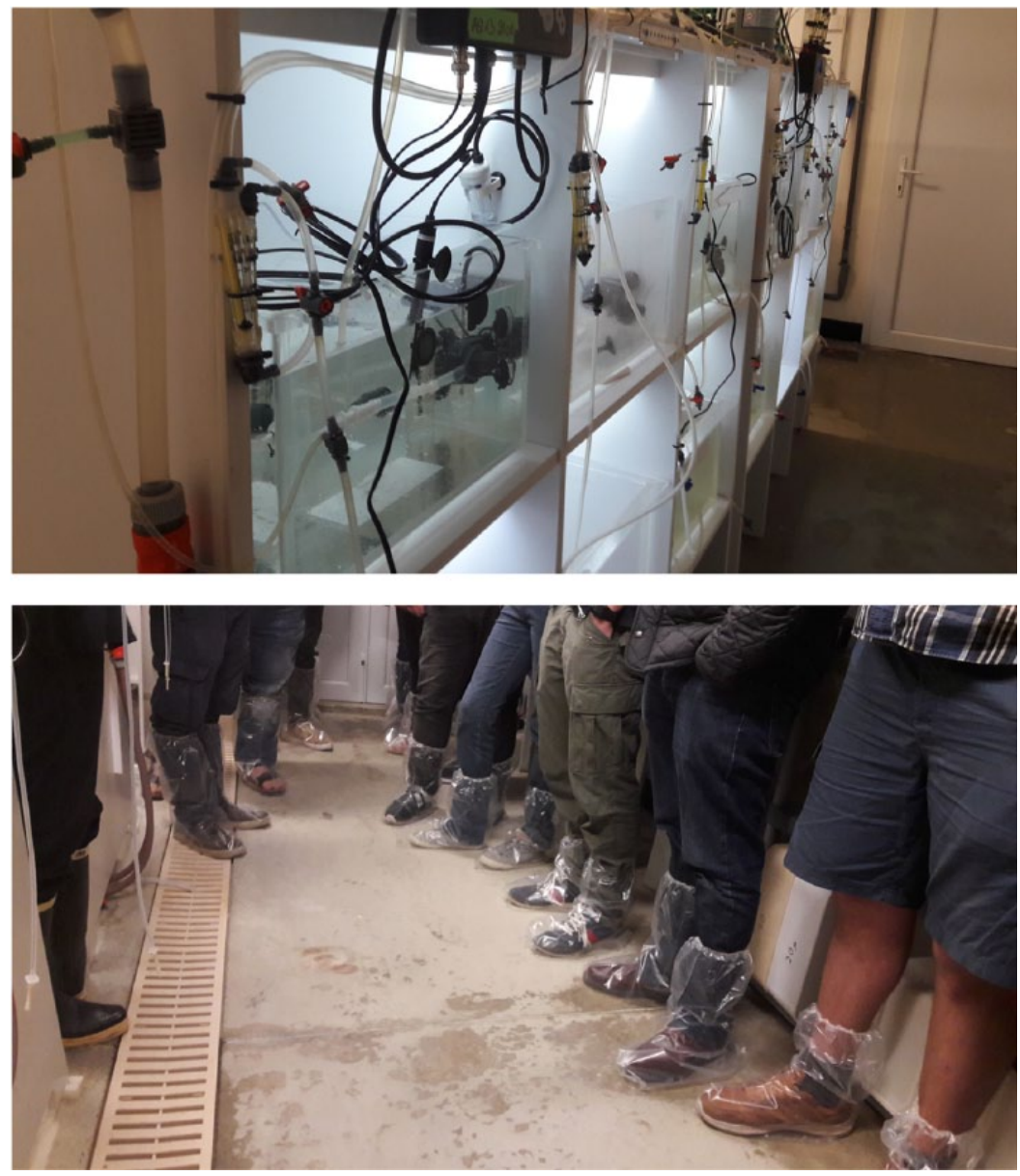

Images 8 et 9 . Visite ethnographique du site expérimental d'Argenton. Laboratoire de Physiologie des Invertébrés - Département de Physiologie des Organismes Marins. Ifremer, LEMAR (UMR 6539).

Crédits : Camille Mazé et Adrien Lambrechts.

ainsi le statut « d'espèce invasive ». Ces deux hypothèses ont des conséquences majeures en matière de décision et de choix de gestion de la baie. Si les nutriments, qui proviennent des activités agricoles, industrielles et urbaines sont le principal facteur de contrôle, il devient dès lors primordial de transformer les infrastructures liées au traitement de l'eau (stations d'épuration) et au-delà, de vérifier l'impact des activités anthropiques sur les bassins versants. Si à l'inverse, on associe le contrôle de la biomasse phytoplanctonique à la présence du mollusque « envahisseur », la question de sa maîtrise se pose (contrôle de l'espèce, éradication), ayant entre autres conséquences, en termes de gestion de l'environnement, une moindre pression sur la nécessité de réduire les apports de nutriments et donc de modifier les infrastructures liées au traitement de l'eau et l'impact des 


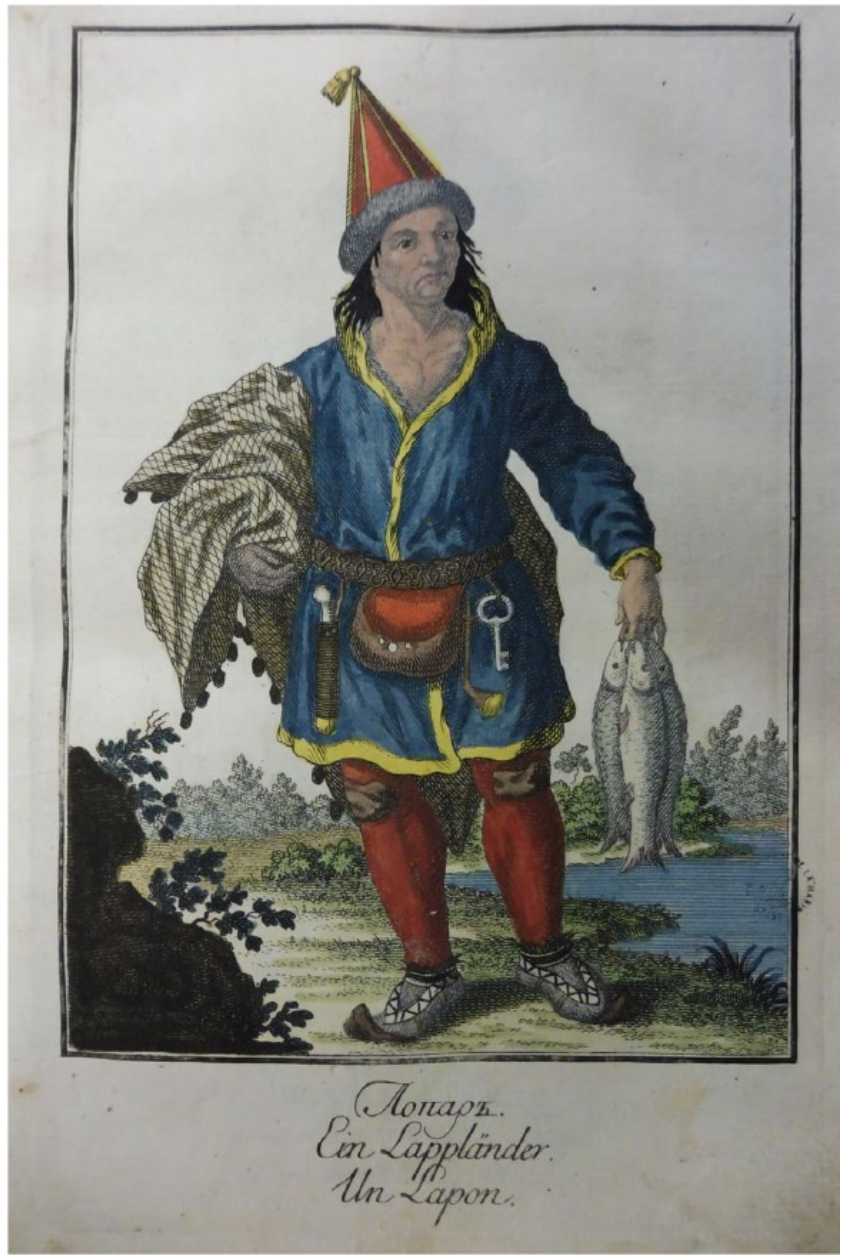

Image 10. Un Lapon, d'après I. I. Georgui, I776.

Credit : ( ) Service Historique de la Défense de Brest, fonds bibliothèque de l'Académie de Marine (R4534).

activités humaines. Cette transformation est néanmoins déjà en cours comme nous le verrons plus avant.

Pour plonger au cœur de cette controverse qui place l'ethnographe à l'interface de la science et du politique, nous nous sommes rendus dans chacun des deux laboratoires où ont émergé et où sont testées ces hypothèses, ainsi qu'au Conseil gouvernemental du Delta du Scramento (Delta Stewardship Council). Des entretiens ont été réalisés avec les chercheurs ainsi qu'avec le chef (Lead Scientist) du Delta Science Program, le Dr. Cliff Dahm.

Le biogéochimiste de l'équipe, O.R., connaît bien les deux scientifiques, Richard Dugdale (Dick) et James Cloern (Jim). Au-delà d'être des collègues, ils sont amis de longue date. Les deux hommes, âgés, à l'image de Dick (84 ans), représentent pour lui 
deux chercheurs incontournables dans leur domaine commun de prédilection, intègres, ouverts et toujours enthousiastes, qui ont profondément marqué leur champ disciplinaire voire interdisciplinaire au fil d'une longue carrière. Dick est un biogéochimiste qui a sillonné les océans, il est en quelque sorte le père de la notion de production nouvelle et régénérée, si importante en océanographie. Sa publication sur ce sujet avec J.J. Goering a fait date, en 1967 (Dugdale et Goering, 1967). Son laboratoire a les pieds dans l'eau, au nord de la Baie. Partie intégrante de l'université de San Francisco (SFSU), Dick dirige une équipe de recherche publique. Jim, quant à lui, est écologue, il travaille à Menlo Park, dans la partie sud de la Baie, presque au cœur de la Silicon Valley, à l'United States Geological Survey (USGS), organisme gouvernemental américain qui se consacre aux sciences de la Terre. Jim a énormément travaillé dans les estuaires et en zone côtière, faisant de la baie de San Francisco un véritable laboratoire expérimental qui a beaucoup inspiré les travaux sur le couplage pelagos-benthos en rade de Brest. Sa publication de 2001 (Cloern, 2001), qui propose un nouveau modèle d'eutrophisation, a généré de nombreuses approches plus intégrées du fonctionnement des écosystèmes, incluant de plus en plus l'homme (concept de socio-écosystèmes: voir Redman, Grove et Kuby, 2004).

En 2013, c'est Jim et ses collègues de l'USGS que nous rencontrons d'abord. Il nous explique l'importance de ce petit mollusque (voir image 12) dans le fonctionnement de la baie. Cela parle au biogéchimiste brestois, car c'est l'équivalent de la crépidule en rade de Brest. Un an plus tard, nous rendons visite à Dick au RTC, sur Paradise drive. Il fait déjà nuit quand nous arrivons. Il nous attend, derrière son ordinateur, trop impatient de nous montrer ses courbes de nitrates et d'ammonium. Pour lui, ce n'est pas tant la quantité que la qualité des nutriments qui joue un rôle fondamental dans la biodiversité phytoplanctonique de la baie. Et il nous l'explique longuement, nous parle de ses hypothèses, de tests de son labo, des nombreuses publications prouvant leurs hypothèses. Il entend redorer le blason des différentes formes azotées en tant que facteur principal contrôlant la biodiversité planctonique en baie de San Francisco : «Regardez ce petit organisme, comment voulez-vous qu'il puisse jouer un rôle si important, il est si petit ?! ».

L'approche ethnographique en binôme, dans une démarche qui va au-delà de la traduction, fait ici ses preuves. Elle permet de mieux saisir et d'approfondir au cours des entretiens, soit les hypothèses scientifiques sur le fonctionnement de l'écosystème, soit les intrications entre les connaissances scientifiques et l'utilisation ultérieure de ces connaissances dans le champ du politique et de la gestion des socio-écosystèmes. Nous sommes plongés au cœur de la production de la connaissance sur l'eutrophisation qui circule ensuite dans les sphères de la décision. Que pense-t-il des hypothèses de Jim ? Quelles sont d'après lui, les implications de cette controverse en matière de gestion de la baie? Quels sont leurs liens avec les gestionnaires, les décideurs ? La politiste pose des questions, tant sur les nitrates que sur ces aspects de l'interface science-politique et le regard de Dick pétille : qui sont les coupables ? Lui et sa femme nous en diront ensuite beaucoup plus au restaurant. La discussion s'achève trop tôt. Mais le terrain de la science nous est ouvert.

Il nous fallait à présent comprendre ce que les décideurs et les gestionnaires de la baie font de ces informations contradictoires ? Comment décident-ils en situation de controverse des orientations à donner en matière de gestion de la baie ? Qui décide et comment, 


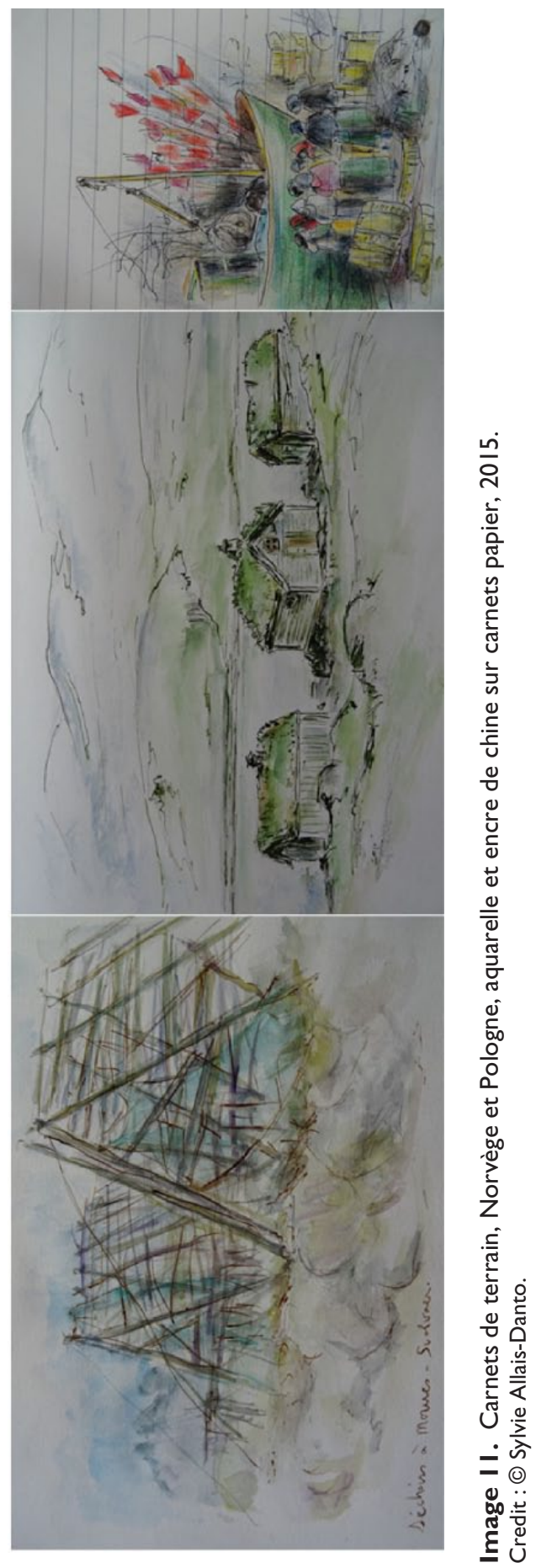




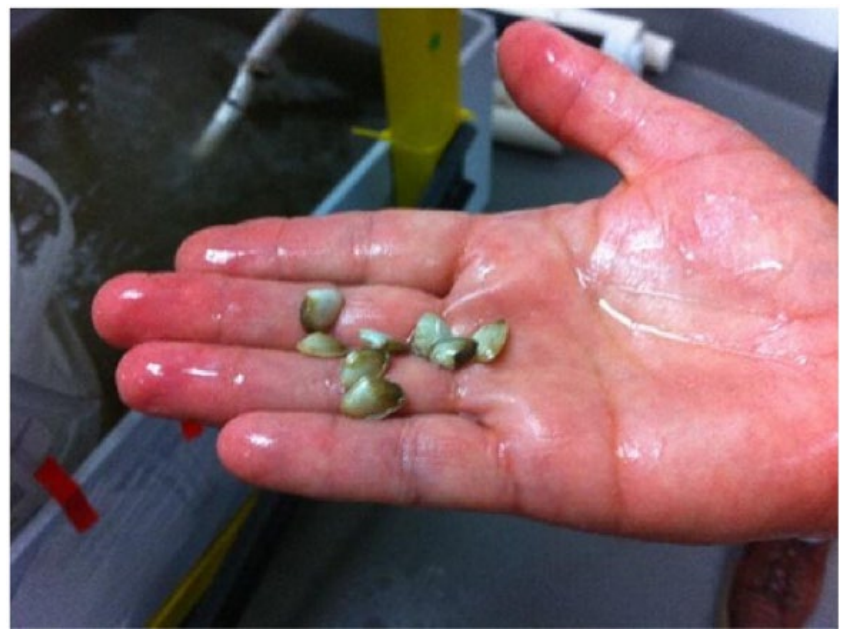

Image I 2. Les « clams » de la baie de San Francisco, 2014.

Credit : Camille Mazé.

de ce qu'il faut faire pour atteindre les objectifs de développement soutenable de ce socio-écosystème sous pression?

L'enquête se poursuit deux ans après ces visites de laboratoire. Les entrevues reprennent. C'est Cliff Dahm qui nous accueille dans son bureau de Sacramento, pour un entretien qui durera plus d'une heure. Cliff est le grand chef du « Delta Science Plan », le bras scientifique du « Delta Stewardship Council ». Il sort d'une réunion avec le Gouverneur de Californie et une quarantaine de personnes, concernant la stratégie de la Californie pour résister à la politique environnementicide que la nouvelle administration Trump ne manquerait pas de lancer... Cliff décrit son organisation comme étant ce qu'on appelle aujourd'hui une " boundary organization », à la frontière entre science et politique. Ils sont scientifiques, lui-même est biogéochimiste, mais ils ne produisent pas de données nouvelles, au sens où ils iraient réaliser des prélèvements d'eau et des carottes de sédiment. Leur rôle est plutôt de réaliser des travaux de synthèse, des états des lieux des connaissances à propos de la baie et de son delta, qu'ils rendent disponibles en organisant des conférences, en publiant des rapports. Il s'agit de porter à la connaissance du public et surtout des décideurs, pour une information d'ordre public. O. R. l'interroge sur la controverse qui oppose les équipes de Jim et Dick. Il raconte que leur façon de gérer cette incertitude a consisté en l'organisation de grandes conférences, tous les deux ans, réunissant les deux équipes, mais faisant également intervenir de nombreux scientifiques d'autres régions des États-Unis (Florida keys, Chesapeake Bay, Great Lakes, Puget Sound) pour apporter un regard extérieur. La question est toujours en débat, mais aujourd'hui, les usines de traitement de l'eau sont déjà en train d'être modifiées. D'un traitement secondaire (rejet de grandes quantités d'ammonium), elles devraient passer en deux étapes à un traitement tertiaire (tout l'ammonium transformé en nitrates), en partie en 2019 et en totalité d'ici 2021. Cette transformation qui consiste en la conversion de l'ammonium en nitrate (nitrification) permet de diminuer le taux d'ammonium dans 


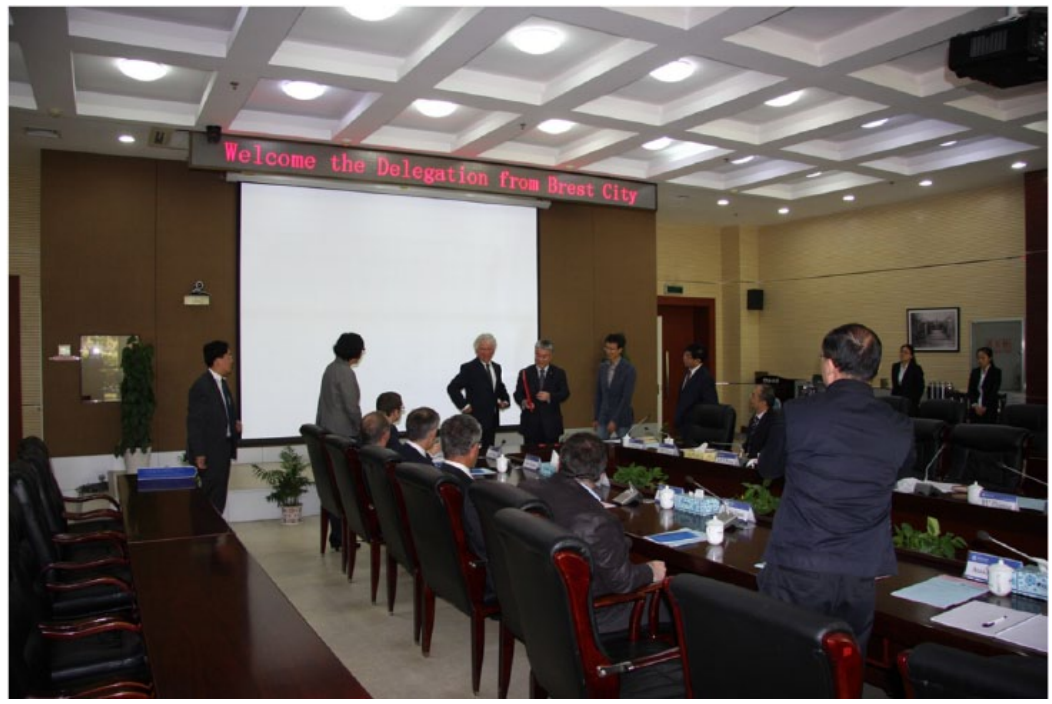

Image 13. Accueil de la délégation brestoise à l'Université océanique de Chine, Qingdao, Mission Jeanne d'Arc mai 2015.

Credit : Camille Mazé.

l'eau, favorisant par là-même le maintien de populations de phytoplanctons sains (diatomées). Nous avons donc affaire à une véritable expérience à l'échelle de la baie, dont les scientifiques vont pouvoir profiter pour tester in vivo leurs hypothèses, en observant sur plusieurs années, la réponse du phytoplancton et de toute la chaîne alimentaire à ces modifications dans la qualité et la quantité des nutriments apportés à la Baie.

L'équipe du LEMAR-ApoliMer continue à enquêter sur ce sujet, mais cette première affaire, complexe, sur le gouvernement de l'interface terre-mer, nous met la puce à l'oreille. Très rapidement, nous voulons creuser cette problématique, interroger des acteurs, comparer avec des cas similaires, comprendre qui fait quoi. Une bonne nouvelle arrive : nous avons la possibilité d'aller en Chine, où le même mal sévit... L'enquête continue, direction l'Asie !

\section{Saison 3 - Tenir le cap : la baie de Jiaozhou}

Carnet de terrain, mai 2015, Baie de Jiaozhou, Qingdao, Chine. «Mai 2015. Nous sommes à Qingdao, dans la province du Shandong qui est jumelée à la région Bretagne, comme membres de la délégation de la Mission Jeanne d'Arc, que nous avons rejoint à Shanghai le soir du samedi 9 mai, avant le transfert pour la ville aux algues vertes.

Ce soir-là, un cocktail était donné en présence de l'Ambassadeur de France en Chine sur le BPC Dixmude (Bâtiment de Projection et de Commandement de la Marine nationale). Nous représentons la recherche brestoise et française, au sein de la délégation venue depuis la région brestoise (voir image 13). Le lendemain matin nous serons transférés pour Qingdao pour y ouvrir notre terrain en présentant notre projet de recherche tri-site. 
Nous sommes venus discuter scientifiquement avec les collègues chinois de la pertinence de notre approche, trouver des partenaires et mesurer la faisabilité de l'enquête sur place ainsi que leur intérêt pour les autres sites d'étude. "

L'observation ethnographique se met en route, reprenant la même méthodologie que lors de l'enquête aux États-Unis. La piste, brouillée, des apports des bassins versants ressort. Lors d'un dîner, nous sommes assis parmi une dizaine de personnes, face au maire de Brest, François Cuillandre et à côté du président et fondateur de la société Olmix, spécialisée dans l'extraction des produits actifs des algues, présente entre autres en France (Bréhan) et en Chine (Qingdao). Olmix finance en CIFRE une thèse en codirection à l'UMR AMURE sur la contribution de l'exploitation des algues vertes à la croissance bleue. Nous aurons la chance d'avoir avec nous Yang-Hi, une doctorante d'origine chinoise, durant la suite du séjour. Elle fait office de traductrice quand les étudiants chinois apprenant le français n'assurent pas ce rôle, mais elle aura été pour nous surtout une informatrice privilégiée. Le terrain chinois est un terrain difficile, qui plus est lorsqu'on y arrive avec le projet d'y développer une anthropologie politique : les rapports politiques, complexes et peut-être plus difficilement saisissables directement qu'en occident sont pourtant fortement sous-jacents au cours des échanges.

Nous allons d'abord exposer notre projet à nos homologues chinois à l'Université océanique de Chine (UOC), au Laboratoire de biogéochimie marine (Key Laboratory of Marine Chemistry Theory and Technology) et nous recevons un excellent accueil du Pr. $\mathrm{Su}$ Mei Liu, une collègue que les chercheurs en biogéochimie de chez nous nous avaient vivement conseillée d'aller voir. Cela a grandement facilité notre entrée sur le terrain des laboratoires. Le Dr. Li Keqiang travaille sur le sujet qui nous anime, l'impact anthropique et la pollution de la baie de Jiaozhou et il interagit avec les autorités publiques pour les conseiller, notamment pour fixer le seuil d'acceptabilité démographique de la baie et alentours. Nos collègues chinois acceptent le principe d'une collaboration (accès aux données, entretiens) mais pointent toutefois, au fur et à mesure de l'échange, la complexité de la nature et les limites de la qualité des données en Chine : certaines sont manquantes ou non disponibles, il existe des « trous dans les données publiques à combler par les scientifiques ». Ils mentionnent « l'urgence écologique et le besoin de gestion intégrée de la baie » ainsi que « le peu d'intérêt jusqu'à présent en Chine pour la question écologique et la biodiversité notamment pour la faune (oiseaux, poissons, mammifères marins) ». Su Mei prend son téléphone et nous organise deux rendez-vous : un le lendemain au département de droit et science politique et l'autre, le surlendemain avec des biologistes halieutes (ressources, pêche).

La réunion protocolaire avec les représentants de l'UOC a lieu devant les membres des volets institutionnels et économiques de la délégation. Il s'agit de discuter avec les académiques de la recherche et de la formation sur la Mer. Il va falloir présenter le projet officiellement devant cette assemblée.

La discussion est ouverte par le Vice-Doyen de Droit et Secrétaire du Parti (Cui Feng), qui parle spontanément de notre projet de recherche, en regard des axes de travail de son laboratoire, mentionnant ainsi son intérêt pour le projet. Noter la double casquette : universitaire et politique. Il présente des pistes en effet assez proches de nos préoccupations : gestion et aménagement des zones côtières, modes de vie traditionnels des pêcheurs et modernisation de la pêche, gouvernance du littoral, etc. Mais il s'offusque lorsque nous 
lui donnons l'information de notre visite au département de droit, de n'avoir pas été averti de notre rencontre à venir l'après-midi avec son Doyen (le rendez-vous avait été organisé par Su Mei la veille par téléphone). Il quitte la salle, manifestement très fâché, en expliquant que ce projet ne l'intéresse pas. Nous venons d'être témoins des dissensions internes existantes dans les rapports politico-scientifiques locaux, où tout est à la fois entremêlé, et extrêmement dichotomique entre la droite ligne du parti et l'ouverture au monde. Au milieu du gué : la question environnementale, tiraillée entre affaire d'Etat et représentation rendue à l'international.

Au même moment, une réunion a lieu au Département des sciences halieutiques, sur le Campus de Hushan, avec l'ingénieur écologue de l'équipe. Le directeur nous présente le contexte écologique de la baie et les interactions entre leur département et les décideurs pour une gestion intégrée de la baie : législation en lien avec les activités d'exploitation des ressources, limitation de la poldérisation, limitation des pollutions industrielles. L'intérêt pour le projet est aussi franc que la veille. Ils acceptent le principe d'une collaboration avec accès aux données et entretiens réalisables en cas d'obtention d'un financement.

L'après-midi, nous nous rendons tout de même au département School of Law and Political Science. Nous souhaitons avoir une présentation de l'état des recherches et de l'état de l'art sur la question de la « gouvernance » de la baie des bassins versants, recueillir leurs points de vue sur la situation écologique et les cadres juridiques et institutionnels, négocier un futur accueil sur le terrain et l'encadrement d'un éventuel doctorant ou postdoctorant dans leur unité de recherche. Ils sont intéressés par le projet et la collaboration.

Une visite de terrain de la baie a lieu juste après, organisée par nos hôtes. Une voiture noire aux vitres teintées nous attend pour nous conduire, pendant plusieurs heures, à travers la ville. Nous roulons sans doute vers la baie mais nous ne la voyons pas. Nous avons l'impression de tourner autour, mais nous sommes dans la ville. Tout notre parcours est urbanisé, nous traversons des zones industrielles, passons devant les usines de General Motors, des individus en bleu de travail, avec des casques blancs, des bottes, à pied ou à mobylette partout. Nous atteignons finalement la baie à la nuit tombante (voir image 14). Nous prenons le pont qui la traverse. Le séjour touche à sa fin. Nous repartons avec un sentiment d'inachevé mais une ouverture de terrain envisageable, à retravailler avec les collègues qui ont l'habitude de ce genre de terrain difficile : les pressions culturelles entre « Orient et Occident » sortent au grand jour, entre les immeubles et les cheminées d'usines qui fument. Les questionnements qui agitent la Chine contemporaine, à michemin entre ouverture mesurée et bonne image donnée à l'étranger nous sautent à la figure. Les jeux politiques internes, entre membres du parti et universitaires ressortent fortement, impactant notre visite. Plus tard, nous découvrirons d'autres terrains, finalement encore plus difficiles. Les joutes politico-internes chinoises lors de notre venue ne sont en réalité que les prémices de ce qui va devenir rapidement notre quotidien sur d'autres terrains plus au nord.

Suivant toutefois quelques pistes intéressantes encore non explorées, les enquêteurs d'ApoliMer se remettent en mouvement, et organisent les trois saisons suivantes, à l'aide de programmes de recherche, de terrains exploratoires, de rencontres, d'actions de formation... La recherche reprend de plus bel. Nous quittons l'Asie pour des contrées plus nordiques, où enquêter peut s'avérer très ardu ! 


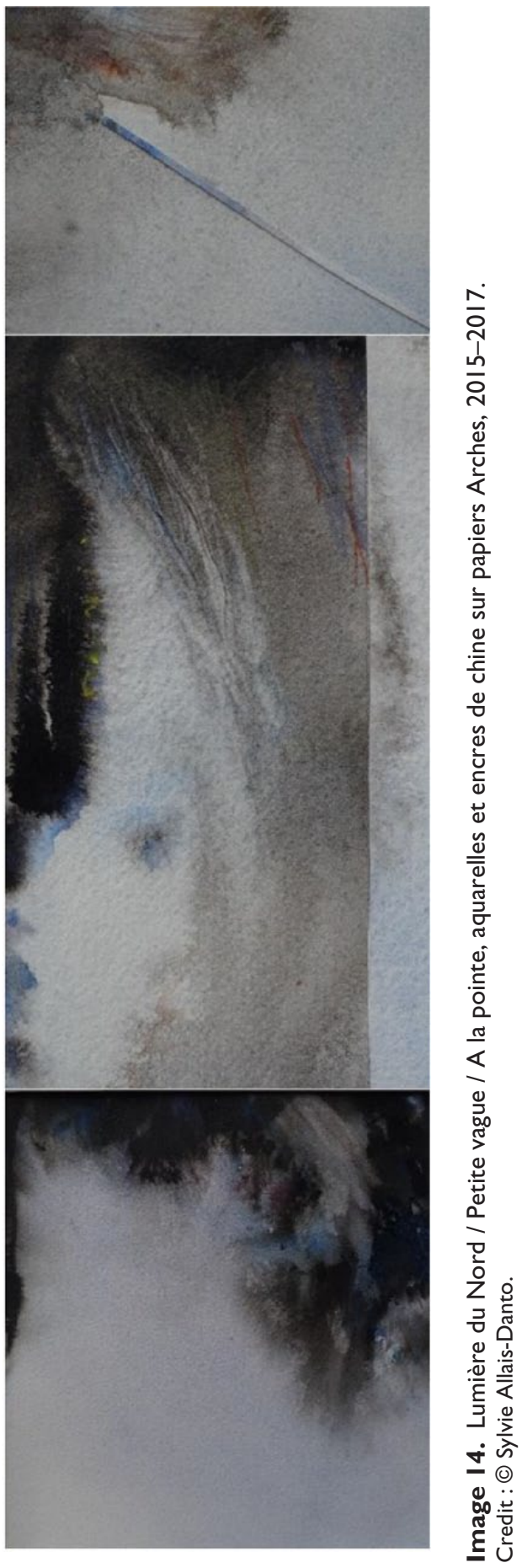




\section{Saisons 4, 5 et 6 (2016-20/8) : barre au septentrion, de la Baltique à Miquelon}

Les saisons suivantes, à partir de l'année 2016, ont été marquées par des enquêtes de terrain « au Nord », au nord de Brest, en Normandie, au nord de l'Europe, sur les pourtours de la mer Baltique, sur le « chemin du Nord », en Norvège, au nord de la Russie, et au nord-ouest de l'Atlantique, sur l'archipel français de Saint-Pierre et Miquelon, tout en poursuivant le travail autour de notre camp de base : la ZABrI.

La notion d'adaptation au changement global a été travaillée sur une partie de ces sites, en regard d'autres terrains situés au Nord comme au Sud (USA, Russie, Groenland, Canada, Inde et Sénégal), notamment grâce au programme de recherche ARTISTICC.[5] L'équipe d'ApoliMer s'est intéressée à deux des sites de ce programme : la ZABrI et la région orientale du delta de la Léna, autour de la ville de Tiksi, en Sibérie (Russie).

Durant deux ans, les enquêtes collectives sur ces terrains ont été prolifiques, avec le renfort d'étudiants (licences, masters, doctorants). Si le site breton est enquêté régulièrement, selon la disponibilité des acteurs, le terrain sibérien nécessite une importante organisation et la programmation de deux missions.

La première mission a lieu en juin 2014, à Yakoutsk (République de Sakha Iakoutie) et a servi de préparation à la seconde, qui s'est déroulée deux mois plus tard à Tiksi, en Sibérie arctique, port russe de fond de baie, florissant par le passé. C.M., anthropologue du politique, s'est alors rendue à Tiksi pendant plusieurs jours en compagnie d'un ethnologue russe, représentant des peuples autochtones du Nord, d'un ingénieur écologue, ornithologue et photographe, et d'une traductrice. Elle y a rencontré de nombreux acteurs de la région (météorologue, chercheurs, directrice de l'un des derniers kolkhozes de pêche, pêcheurs, marins, enseignants, agents de la réserve naturelle nationale de l'embouchure de la Lena, vétérinaire des élevages de rennes, etc.) sous un important contrôle militaire. L'équipe s'est aussi rendue sur la presqu'île de Bykovskiy (République de Sakha), à l'extrémité orientale du vaste delta de la Léna, à proximité de la réserve naturelle sud du delta. Nous logeons à notre arrivée dans une cabane vétuste, réservée aux travailleurs qui se rendent sur l'île pour des tâches de maintenance technique. Du poisson séché repose dans un sac plastique sur le vieux poêle, à proximité des sommiers à ressort, des matelas de fortune et des couvertures de laine. Différents matériaux ont été acquis (enregistrements d'interviews, bibliographie, photographies, etc.), dont certains font l'effet d'une bombe : le cimetière, côtier, tombe à la mer, à cause de la fonte du permafrost (voir image 15). Le trait de côte ne tient plus, les cercueils glissent, rejoignant les lais et relais, puis disparaissent à la première tempête, lorsque la mer de Laptev se déchaîne.[6] Le changement climatique est à l'œuvre. Le désœuvrement social également. L'armée russe, pourtant si présente à Tiksi, n'intervient pas. Les représentants de la communauté veulent en appeler à l'aide internationale.

En parallèle, des missions d'exploration sont mises en place à partir de 2015-2016 autour de la mer Baltique, ciblant certains territoires en particuliers : les côtes baltes allemandes (Länder du Schleswig-Holstein et du Mecklenburg-Vorpommern), Pomorze (Poméranie polonaise), la Courlande (Lettonie), et les golfes de Riga et de Finlande, la Carélie (Russie) et l'Ingrie (Russie). L'adaptation aux changements, environnementaux 


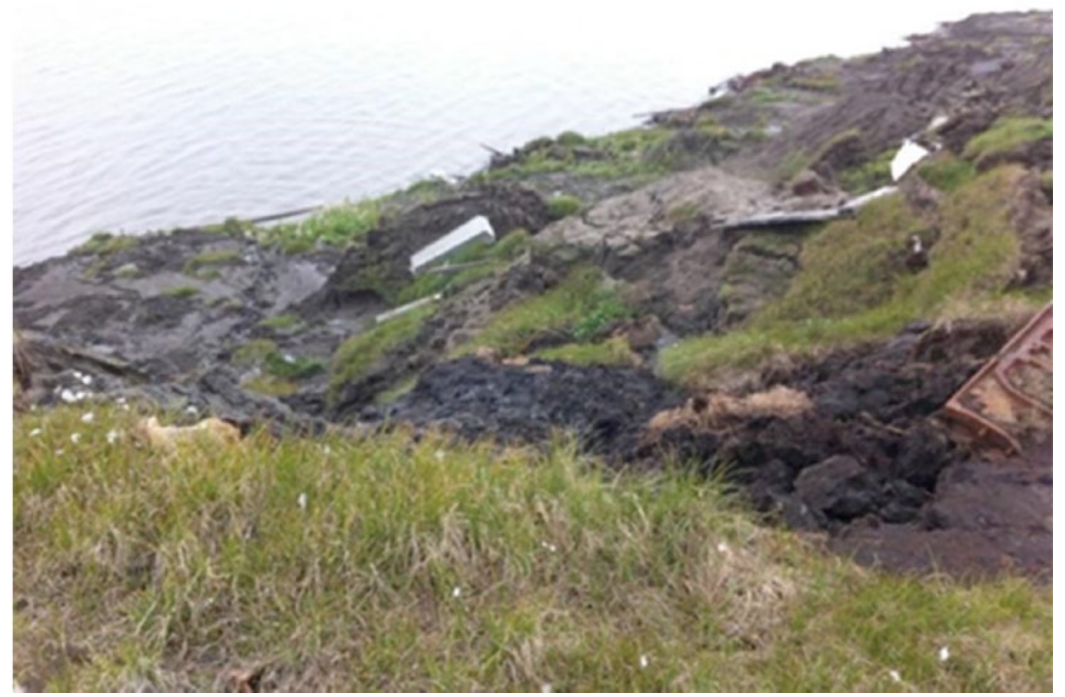

Image I5. Cimetière de Bykovsky près de Tiksi (Sibérie), 2014.

Credit : Camille Mazé.

comme socio-économiques, des communautés côtières, y est aussi questionnée, notamment sous le prisme des trajectoires des pays post-socialistes et post-soviétiques.

Carnet de terrain, janvier 20 I 6, île de Kihnu, golfe de Riga, Estonie. " Nous sommes sur l'île depuis déjà trois jours. En plein mois de janvier. $-20^{\circ} \mathrm{C}$ au bord de l'eau. Grand vent. La glace devrait bientôt être assez épaisse pour que les services locaux de l'équipement stoppent la navigation maritime, et ouvrent la route de 8 kilomètres qui relie l'île au continent pendant l'hiver, quand le golfe est suffisamment gelé (voir image 16). La directrice du centre culturel nous loge dans son sauna, de l'autre côté de la cour par rapport à sa ferme, $[\ldots]$ et cet après-midi-là, elle propose de nous déposer au musée local, face à l'église. Nous acceptons. Après quelques minutes de pick-up, et un dérapage sur la neige qui recouvre le parking de l'église, nous descendons pour visiter le musée. Nous sommes les seuls visiteurs de l'après-midi. Après une découverte des collections sur la chasse au phoque et la pêche au hareng, accompagnée d'un thé revigorant, le téléphone de la gardienne sonne. Notre logeuse, la directrice, nous convie à la rejoindre à la ferme de X, car un anniversaire y est fêté. La gardienne nous montre sur une carte de l'île où se trouve la-dite ferme, et après une demi-heure de marche sur les petites routes de Kihnu, nous arrivons, transis, chez X. Comme le veut la superstition finno-ougrienne, la porte nous est ouverte, mais aucune salutation n'a lieu sur le seuil, pour ne pas porter malheur. Enfin rentrés dans la maison, nous sommes dirigés vers la cuisine pour faire les présentations. L'anniversaire du jour est celui du propriétaire, marin-pêcheur de profession. On nous sert une vodka et nous propose du poisson (du vimbe). Le pêcheur ouvre la fenêtre, et se 


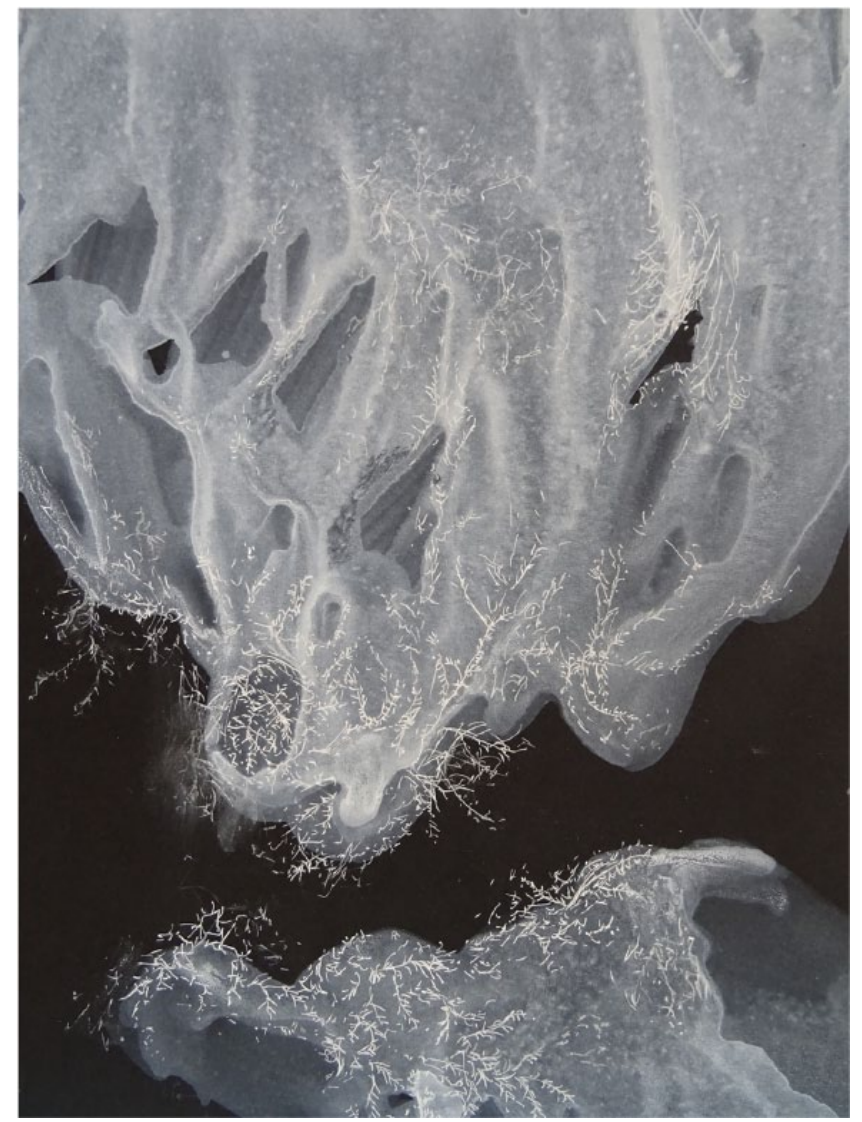

Image 16. Glaciation, aquarelle et crayon sur papier, 2015.

Credit : () Sylvie Allais-Danto.

saisi d'un poisson congelé par les températures hivernales de la Baltique, directement posé sur le rebord de la fenêtre. Il le vide et lève les filets, puis laisse à la directrice le soin de terminer le travail : enlever les écailles, et les arrêtes, puis le découper finement sur une planche, et le servir, cru. Parallèlement au travail féminin de préparation du vimbe, le pêcheur reçoit en cadeau un autre poisson, lui aussi gelé, un brochet. La directrice s'assied à la table, distribue le poisson, et tandis qu'un des hommes présents se réveille laborieusement pour jouer de l'accordéon, elle se met à nous expliquer la signification de certains rêves pour les pêcheurs : " sur l'île, pour les hommes, rêver une nuit qu'ils font l'amour vigoureusement à leur femme leur fait dire que la pêche sera excellente le lendemain matin ». Puis, très vite change de sujet : « la chasse au phoque est interdite depuis 1980. Le problème, c'est qu'elle est populaire ici à Kihnu. Alors il y a trois ans, le gouvernement l'a à nouveau autorisée, sous la pression des gens de l'île. Mais les pêcheurs n'ont pas de licence pour chasser. Donc on reste dans l'illégalité dans un sens » (voir image 17). 


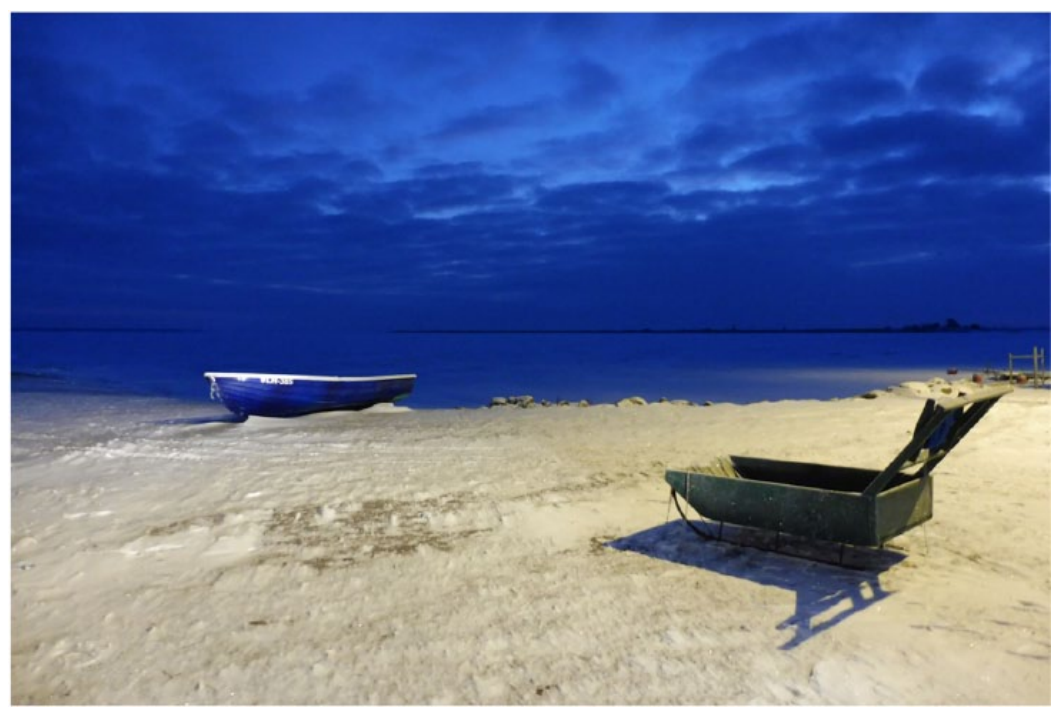

Image I7. Le départ à la pêche sur la glace approche, île de Kihnu, Estonie, 2016. Credit : Anatole Danto.

Son 3 : DakhaBrakha, Yanky, Ethno-mécanique [ДахаБраха, Янки, Этномеханика], 2010.

https://www.youtube.com/watch?v=qo0PHIH7zs0

Carnet de terrain, octobre 20 I 7, île de Saint-Pierre, Saint-Pierre et Miquelon, France. «Arrivée à l'heure avec le vol d'Air Saint-Pierre le 7 octobre 2017. Après le contrôle de la police aux frontières et des douanes, nous emménagerons au gîte. L'équipe sciences de la nature est repartie, sa mission a pris fin il y a quelques semaines.

Le Navire Océanographique Antea de $35 \mathrm{~m}$, armé par Genavir, a mené une campagne océanographique autour de l'archipel de mi-août à mi-septembre (campagne Saint Pierre et Miquelon 2017) (voir image 18). Une trentaine de chercheurs se sont relayés pour travailler sur les facteurs physiques (étude des courants, de la salinité et des températures autour de l'archipel), la biodiversité benthique (inventaire de la ressource : coquillages, crabes...), la biodiversité phytoplanctonique (inventaire des cellules phytoplanctoniques, base de la chaîne alimentaire), étude des bivalves (principalement les pétoncles et les palourdes noires). La ministre des Outre-mer a visité le catamaran jeudi 11 août.

Au cours de cette mission, les plongeurs scientifiques du LEMAR ont sondé les fonds de l'archipel, profitant de l'aide de Herlé Goraguer, le délégué de l'Ifremer (Institut français de recherche pour l'exploitation de la mer) à Saint-Pierre et du support logistique du club de plongée de Saint-Pierre et Miquelon. Je viens rejoindre Camille qui est présente depuis le 20 août sur le terrain et réalise plusieurs séries d'entretiens, avec Olivier en sociologie politique des sciences, et Adrien, ingénieur écologue spécialisé dans l'évaluation de l'impact environnemental, pour décrypter le processus de décision concernant la gestion de Saint-Pierre et Miquelon (SPM) et notamment la prise en compte 


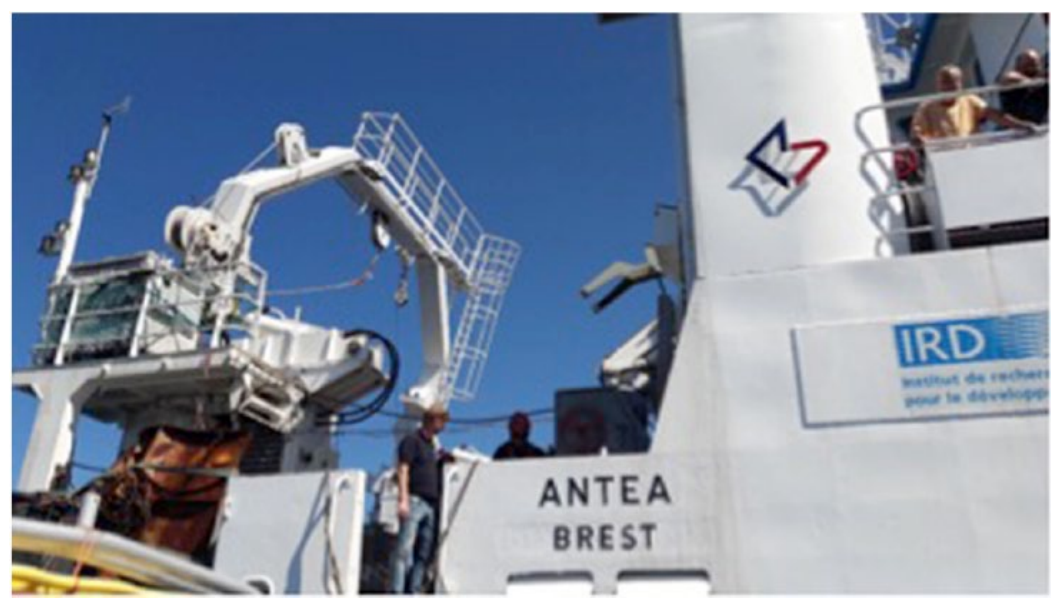

Image 18. Campagne scientifique « SPM 2017 »-Antéa, août 2017.

Crédit : Camille Mazé.

des enjeux environnementaux par rapport à d'autres considérations (économiques, sociales, stratégiques, symboliques). Cette petite équipe interdisciplinaire interagit à la fois avec les chercheurs en sciences de la nature et les élus, les gestionnaires et les acteurs économiques de SPM, au niveau local (Maire), territorial (Collectivité territoriale) et national (Ministre des outre-mers venus avec son staff pour le lancement des Assises de l'Outre-Mer en octobre 2017, présence à ce moment-là de l'armateur Le Garrec). »

Elle va de l'observation des « expérimentations » à terre et en mer, aux débriefings nocturnes des océanographes jusqu'au bureau des maires, président de la collectivité territoriale et Préfet, pour comprendre le processus de production et d'utilisation des connaissances (voir image 19). Nous analysons la chaîne dans son ensemble : depuis ce qui pose problème pour les scientifiques jusqu'à la construction et au traitement qui en est fait (Blemer et Glavonic, 2013). Les entretiens s'enchainent, ainsi que les discussions informelles avec les habitants, décideurs, scientifiques de l'archipel. Tout est consigné dans des carnets, observé et noté. Les membres de l'équipe recoupent les informations entre elles. Le focus est mis sur l'analyse de la trajectoire socio-écosystémique de SPM, depuis le crash de la pêche à la morue jusqu'à l'exploitation industrielle du concombre de mer (holothurie).

Ancien département d'outre-mer, aujourd'hui passé au statut plus autonome de collectivité d'outre-mer, Saint-Pierre et Miquelon est l'unique terre française d'Amérique du Nord. Situé à 25 kilomètre de Terre-Neuve, l'archipel est constitué de deux îles principales Saint-Pierre et Miquelon-Langlade ainsi que d'une huitaine de petits ilots inhabités. Sa localisation particulière en fait une ressource stratégique pour l'Etat français pour des raisons géopolitique et économique.

Depuis le moratoire canadien de 1992 réduisant de manière drastique les quotas alloués à la pêche à la morue, la rendant de facto presque interdite, le secteur halieutique s'est effondré. Celui-ci étant la principale source de revenus de l'archipel, l'économie locale s'est rapidement trouvée obligée de s'adapter. Aujourd'hui, elle fonctionne 


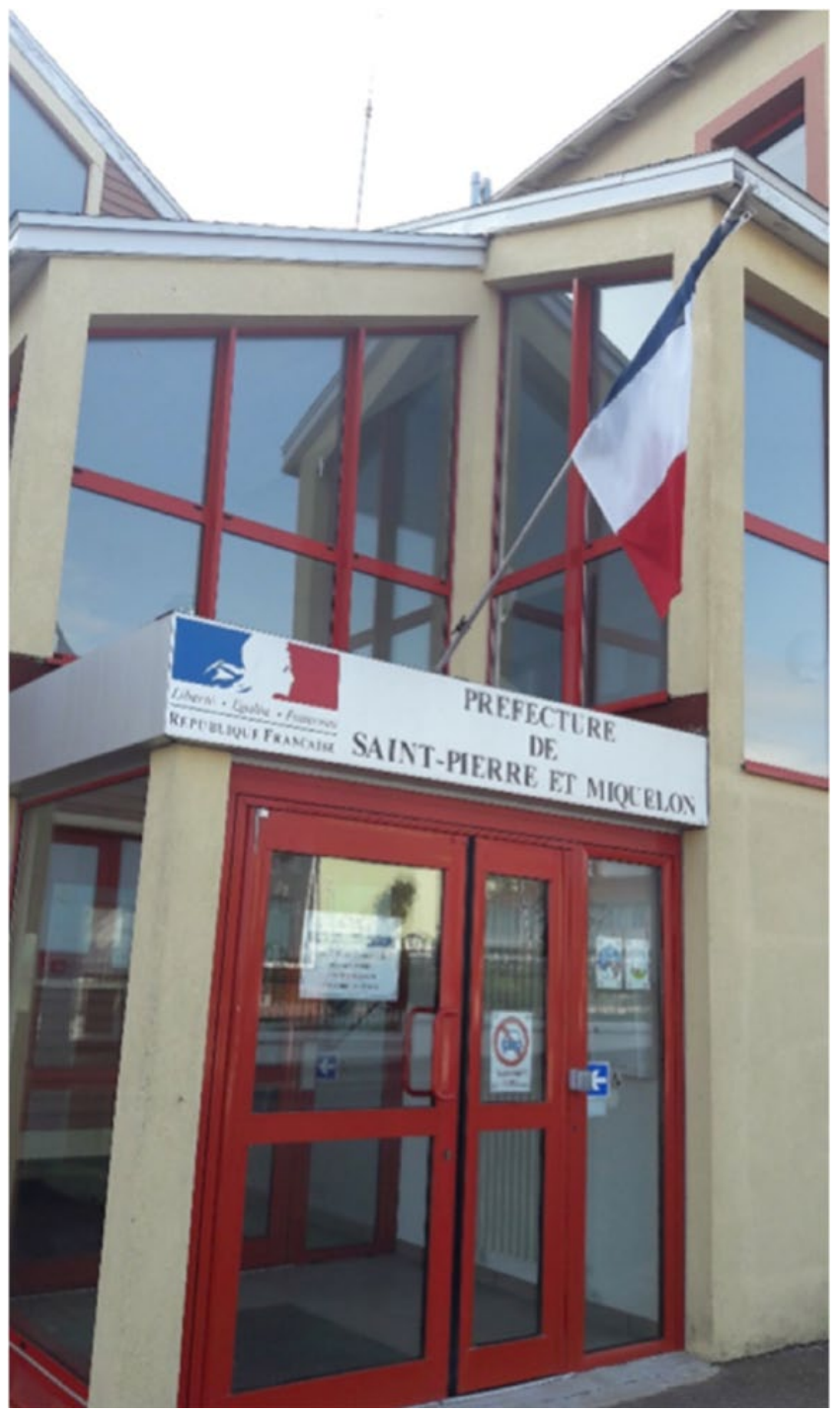

Image 19. Préfecture à Saint-Pierre et Miquelon. Aout 2017.

Crédit : Camille Mazé.

majoritairement grâce aux aides de l'Etat et aux fonds européens, ainsi qu'à un système fiscal propre à Saint-Pierre inauguré en 1946. De la Doris qui a révolutionné la pêche à la morue sur les bancs de Terre-Neuve au Keravel, bateau le plus polyvalent de SPM dans sa catégorie (chalutier, ligneur, palangrier hydraulique mais aussi dragueur), jusqu'à la nouvelle filière d'exploitation du « concombre de mer », très prisée sur le marché asiatique, pêchée au large de l'archipel, traitée sur le territoire puis au Canada avant d'être exportée (voir image 20). 


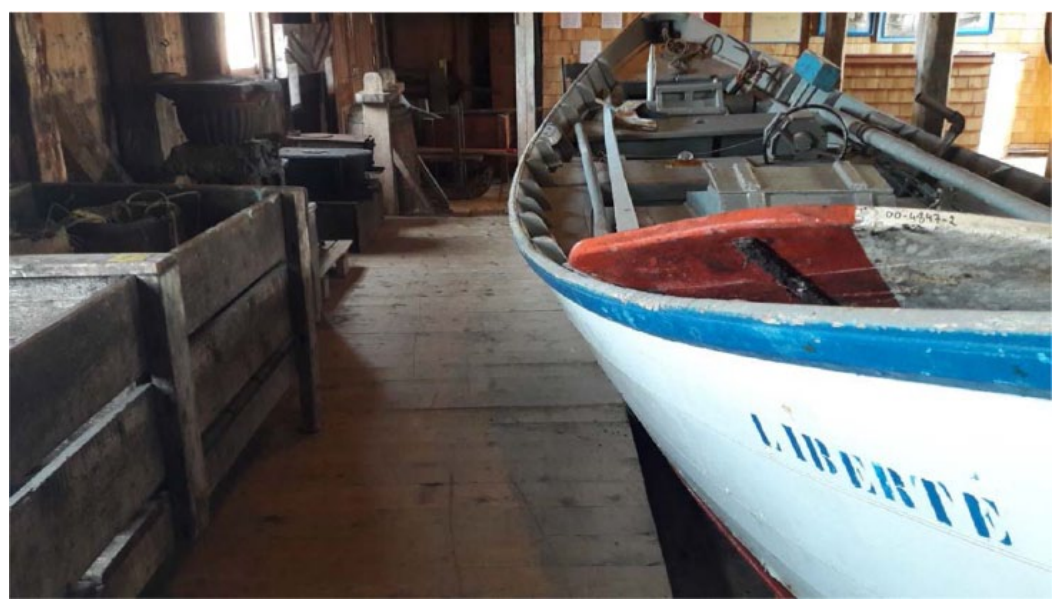

Image 20. Evolution de la pêche à Saint-Pierre et Miquelon.

Crédit : Camille Mazé.

Cette dépendance économique vis-à-vis de la métropole est particulièrement mal vécue à Saint-Pierre et Miquelon qui craint en permanence une réduction de ces aides indispensables à sa survie provoquant ainsi une véritable volonté des pouvoirs publics de promouvoir le secteur du tourisme ainsi que le développement numérique et des services au sein de ce territoire.

La raison d'être historique de Saint-Pierre et Miquelon étant la pêche (voir image 21), sa progressive extinction met l'archipel face à un douloureux questionnement sur son identité entrainant des rapports parfois conflictuels avec la métropole ce qui est renforcé par son isolement et la distance. L'activité halieutique y est aujourd'hui anecdotique malgré quelques courts épisodes d'abondance avec le pétoncle ou le crabe de mer, mais ceux-ci ont rapidement cessés du fait d'une pêche déraisonnée de ces espèces entrainant une quasi-disparition des stocks. Le phénomène se reproduit avec le concombre de mer, très populaire sur le marché asiatique, qui est pêché en quantité importante sans données scientifiques suffisantes concernant le renouvellement du stock.

Son 4 : Marie Boine Persen, Gula Gula, 1989.

https://www.youtube.com/watch?v=k4s8ZATQeBI

\section{Pour une approche éco-politiste de la mer}

L'analyse critique - au sens constructif du terme - de la notion très en vague (à la fois à la mode mais floue) de « gouvernance de la mer » (Mazé et al., 2017) amène l'équipe à naviguer à la fois dans l'espace social et dans l'espace naturel - socialisé - : en gravitant des lieux de production des normes et des instruments de « gouvernance » liés à la gestion de l'environnement marin (arènes de décision politique, lieux de mobilisations 
collectives, de luttes, de résistances, de protestation ou d'investissement pour une cause), aux lieux de production des connaissances scientifiques et d'expertise (laboratoires, bureaux d'étude), en visitant les terres d'émergence d'autre formes de savoirs (communautés autochtones, projets artistiques) et en s'immergeant au cœur même du fonctionnement et de l'évolution des écosystèmes marins et côtiers.

Les observations ethnographiques, dont seulement quelques extraits ont été ici présentés, constituent un matériau de premier choix pour conduire notre analyse critique : le

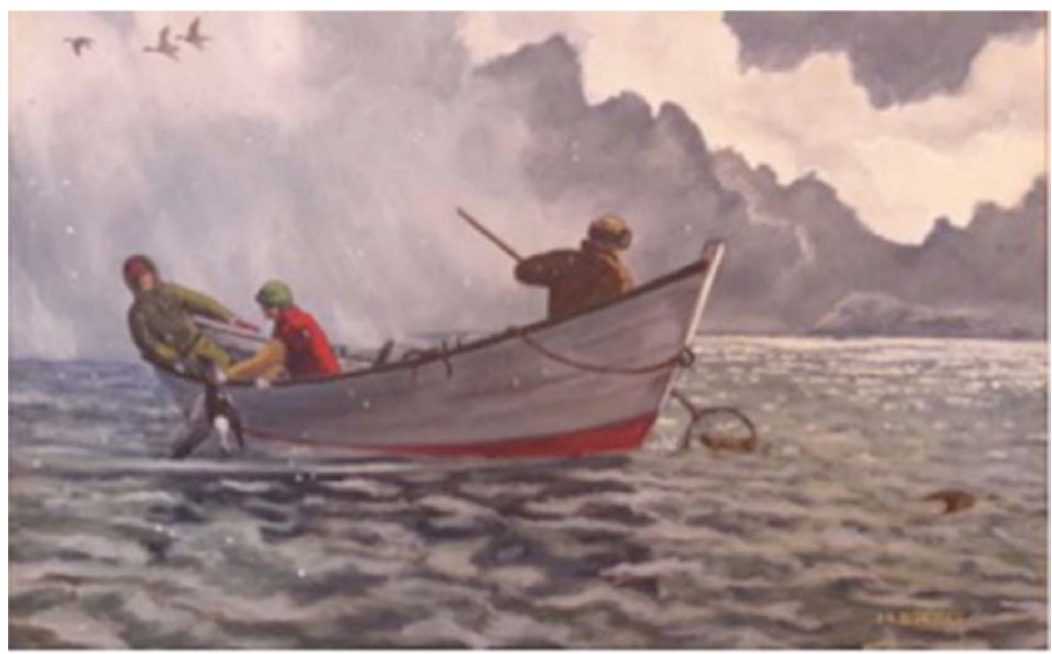

Image 21. Peinture photographiée dans les locaux de la Collectivité territoriale de SaintPierre et Miquelon, août 2017.

Credit : Camille Mazé.

groupe de recherche s'attache à décrypter les modes de gouvernement de la Mer, notamment dans le contexte du changement à l'échelle du globe, à l'aide d'enquêtes auprès d'une multitude d'acteurs. Ces matériaux empiriques, issus de la pratique de terrain, permettent une mise en exergue des problématiques politiques qui agitent la mer aujourd'hui, plus que jamais prise dans des circulations et des transferts au sein des réseaux de " gouvernance » du local au global : scientifiques, décideurs, communautés, artistes, acteurs industriels, militants écologistes, naturalistes, usagers des littoraux, touristes, etc.). Les alliances et tensions économico-politiques et scientifiques comme en Chine ou aux Etats-Unis sur fond d'innovation technologique, les enjeux liés à l'exploitation / commercialisation des ressources marines, les pressions pour le maintien de la souveraineté nationale dans le cadre des zones économiques exclusives et les négociations sur la haute mer (Biodiversité audelà des juridictions nationales, BAJN) de l'échelle nationale aux Nations-Unies, les relations entre Etat et acteurs privés (industrie de la pêche, ONG environnementalistes) autour des créations d'aires marines protégées XXL, les interactions science / décision / industrie dans le nouveau cadre de gestion dite adaptive des socio-écosystèmes marins qui tranchent de manière cinglante avec la réalité des formes de criminalité en mer (piraterie), les conflits 
géopolitiques et les migrations climatiques et environnementales constituent autant de sujets d'enquête et de terrain d'investigation en cours ou futures pour les chercheurs impliqués dans la démarche d'enquête d'ApoliMer.

http://umap.openstreetmap.fr/fr/map/apolimer_135822\#2/4.2/50.6

Carte 1 : interactive des activités d'ApoliMer, 2018. Anatole Danto.

Son 5 : Maud Geffray, Polaar, 2017.

https://www.youtube.com/watch?v=HkX_jR00JGU

\section{Remerciements}

Les auteurs remercient le LEMAR (UMR 6539), l'INEE (CNRS) pour le soutien apporté au RTPi ApoliMer, la chaire TMAP (IEP Rennes) et le Labex Mer, ainsi que la Mission pour l'Interdisciplinarité du CNRS qui supporte le projet SPA (Savoir Pouvoir Avoir) coordonné par C. Mazé. La Zone-atelier Brest Iroise $(\mathrm{ZaBrI})$ et le réseau des zones-ateliers (LTSER) nous apportent également un cadre privilégié pour mettre notre démarche en œuvre sur la durée. Que soit ici aussi chaleureusement remerciés la Maison Julien Gracq à Saint-Florent-le-Vieil et Sylvie Danto pour ses œuvres. Mention spéciale pour l'ensemble des enquêtés et des enquêteurs (étudiants de licence, master et doctorat).

\section{Financements}

L'INEE (CNRS), la chaire TMAP (IEP Rennes) et le Labex Mer co-financent la thèse d'Anatole Danto sur « la gouvernance du terre-mer » codirigée par R. Pasquier et C. Mazé.

\section{Notes}

1 Au premier janvier 2018, après cinq années d'existence et de maturation, ApoliMer a été labélisé Réseau thématique pluridisciplinaire international par l'Institut national écologieenvironnement du CNRS: http://www.cnrs.fr/inee/recherche/actionsincitatives-RTPiApolimer.htm

2 Sur cette thématique, nous renvoyons notamment aux écrits du socio-anthropologue Tarik Dahou (IRD / PALOC, membre d'ApoliMer).

3 Concept très en vogue qui tente une opération cognitive de reconnexion des relations Homme / Nature et qui oublie trop souvent de restituer à cette dichotomie socialement construite sa profondeur historique et sa situation occidentale.

4 Les fonds bibliothèque de l'Académie de Marine sont conservés à Brest, au sein du Service Historique de la Défense, avec lequel nous sommes amenés à collaborer sur de nombreux sujets.

5 Projet ARTISTICC ((Adaptation Research a Transdisciplinary Community and Policy Centred Approach), Belmont Forum, 2014-2017.

6 Plus de photographies de la mission sont visibles sur le site de l'observatoire photographique des pôles : http://observatoirephotographiquedespoles.org/photos-gallery/ expedition-a-tiksi-bulunsky-ulus-republique-de-sakha-federation-de-russie/

\section{ORCID iD}

Anatole Danto (iD) https://orcid.org/0000-0003-2383-9143 


\section{Références bibliographiques}

Adelle C and Weiland S (2012) Policy assessment: the state of the art. Impact Assessment and Project Appraisal 30(1): 25-33.

Bremer S and Glavovic B (2013) Mobilizing knowledge for coastal governance: re-framing the science-policy interface for integrated coastal management. Coastal Management 41(1): 39-56.

Christie P (2011) Creating space for interdisciplinary marine and coastal research: five dilemmas and suggested resolutions. Environmental Conservation 38(2): 172-186.

Cloern JE (2001) Our evolving conceptual model of the coastal eutrophication problem. Marine Ecology Progress Series 210: 223-253.

Dugdale RC, Goering JJ (1967) Uptake of new and regenerated forms of nitrogen in primary productivity. Limnology and Oceanography 12(2): 196-206.

Georgui II, [Георги, И. И.] (1776) Description de toutes les nations de l'Empire de Russe, où l'on expose leurs mours, religions, usages, usages, habitations, habillemens et autres particularités remarquables. Première collection qui contient les nations d'origine finnoise.

Jasanoff S (1990) The fifth branch: Science advisers as policymakers. London: Harvard University Press.

Kates RW (2011) What kind of a science is sustainability science? P.N.A.S. 108: 1949-1945.

Marcus GE (1995) Ethnography in/of the World System: the emergence of multi-sited ethnography. Annual Review of Anthropology 24(1): 95-117.

Mazé C, et al. (2017) Knowledge and power in integrated coastal management: for a political anthropology of the sea combined with the sciences of marine environment. Comptes Rendus Geoscience 349 (6-7): 359-368.

Mooney HA, Duraiappah A and Larigauderie A (2013) Evolution of natural and social science interactions in global change research programs. P.N.A.S. 110: 3665-3672.

Ragueneau $\mathrm{O}$ et al. (2018) The impossible sustainability of the Bay of Brest? Fifty years of ecosystem changes, interdisciplinary knowledge construction and key questions at the sciencepolicy-community interface. Frontiers in Marine Science 5(124).

Redman CL, Grove JM and Kuby LH (2004) Integrating social science into the long-term ecological research (LTER) network: social dimensions of ecological change and ecological dimensions of social change. Ecosystems 7(2): 161-171.

\section{Musique}

Son 1 : Niels Petter Molvær, Solid ether, 2000.

https://www.youtube.com/watch?v=E2GQbt_OL-Y

Son 2 : Terje Isungset et Lena Nymark, Glacier recording, 2014.

https://www.youtube.com/watch?v=QosfSaE0q7c\&list=RDwno0YKp1bGQ\&index=4

Son 3 : DakhaBrakha, Yanky, Ethno-mécanique [ДахаБраха, Янки, Этномеханика], 2010. https://www.youtube.com/watch?v=qo0PHIH7zs0

Son 4 : Marie Boine Persen, Gula Gula, 1989.

https://www.youtube.com/watch?v=k4s8ZATQeBI

Son 5 : Maud Geffray, Polaar, 2017.

https://www.youtube.com/watch?v=HkX_jR00JGU

\section{Author biographies}

Depuis 2016, Anatole Danto a entamé une thèse au CNRS en sciences sociales sur la gouvernance du terre-mer, analysant des cas français et allemands. Diplômé de masters en anthropologie, histoire maritime et expertise et gestion de l'environnement littoral, ses recherches portent 
principalement sur les dynamiques des sociétés de pêcheurs face aux changements politiques, socio-économiques et environnementaux en Europe du nord-ouest. Il est membre du réseau culturel de la convention Ramsar, et représentant français au sein du groupe de travail 'history of fish and fisheries' du Conseil International d'Exploitation de la Mer.

Camille Mazé est docteure en Sciences sociales (ENS / EHESS). Elle s'est spécialisée dans l'étude $\mathrm{du}$ fait politique et des relations de pouvoir, d'abord dans le domaine de la Culture puis dans celui de la Nature - le « grand partage » socialement construit et culturellement situé qu'elle explore. Après avoir exercé comme maître de conférences en anthropologie à l'Université de Bretagne occidentale à Brest (2011-2016), Camille Mazé est devenue chargée de recherche en science politique au CNRS. Elle est membre du Laboratoire des sciences de l'environnement marin (LEMAR UMR 6539) où elle se consacre à l'étude des formes de régulation et d'appropriation des mers et des océans dans le contexte du changement global. C'est dans cette perspective qu'elle a fondé et coordonne le réseau de recherche ApoliMer (RTPI CNRS) destiné au développement d'une anthropologie eco-politique de la mer, articulée avec les sciences de la nature et les sciences de l'ingénieur pour repenser et recomposer les relations Homme / Nature en redonnant tout son sens au politique.

Olivier Ragueneau est un biogéochimiste marin avec une forte attraction pour le travail aux interfaces : entre cycles biogéochimiques, entre ces cycles et les organismes marins, entre eaux de surface et sédiments, entre terre et mer, entre disciplines - d'abord au sein des sciences naturelles puis de plus en plus avec les sciences humaines et sociales -, entre la science et la société et à l'interface de la science-politique. Il est aujourd'hui coordinateur de la « Zone Atelier Brest-Iroise » avec Pierre Stéphan, géographe. Son approche vise la transdisciplinarité, dans le but de répondre à plusieurs défis sociétaux imposés par le changement global (eutrophisation, changement climatique, espèces invasives ...), en développant des recherches collaboratives avec différents acteurs de la société civile (ONG, gestionnaires de bassin, enseignants, artistes). La question de l'interface entre science, société et politique est au cœur de ses réflexions, stimulant à la fois la recherche collaborative et les programmes de sciences citoyennes et gardant un regard critique sur cette évolution de la recherche vers la soutenabilité, à travers la sociologie politique des sciences au sein d'ApoliMer. 